\title{
Damnacanthal: a promising compound as a medicinal anthraquinone
}

\begin{abstract}
The Noni fruit, or scientifically known as Morinda citrifolia can be found in various parts of the world, especially in the pacific region. It is a small evergreen bushy-like tree originated from the Rubiaceae family. The plant has been used by polynesians as a medicinal herb for more than 2000 years. A substantial amount of phytochemicals can be found in the roots of this plant. Among all, damnacanthal has been found to be the most interesting, versatile and potent compound. Damnacanthal or chemically known as, 3- hydroxy-1methoxyanthraquinone-2-caboxaldehyde (C16H10O5), appears as pale yellow crystals with a melting point of $210-211{ }^{\circ} \mathrm{C}$. This compound is of particular interest due to its striking pharmacological properties. Damnacanthal was shown to inhibit the oncogene Ras, p56lck tyrosine kinase, NF-KB pathway and induce apoptosis in vitro. This review aims to discuss the biological properties of damnacanthal, specifically on its anti-cancer activity that has been reported.
\end{abstract}

Keyword: Anti-cancer; Anti-inflammation; Damnacanthal; Morinda citrifolia; Noni; Pharmacology 